\title{
Results of Instrumented Posterolateral Fusion in Treatment of Lumbar Spondylolisthesis with and without Segmental Kyphosis: A Retrospective Investigation
}

\author{
Szu-Yuan Chen, Meng-Ling Lu, Chi-Chien Niu, Tsung-Ting Tsai, Jen-Chung Liao, Lih-Huei Chen, \\ Wen-Jer Chen
}

Background: Treatment by posterolateral fusion (PLF) with pedicle-screw instrumentation can be unsuccessful in one-segment and low-grade lumbar spondylolisthesis. Segmental kyphosis, either rigid or dynamic, was hypothesized to be one of the factors interfering with the fusion results.

Methods: $\quad$ From 2004 to 2005, 239 patients with single-segment and low-grade spondylolisthesis were recruited and divided into two groups: Group 1 consisting of 129 patients without segmental kyphosis and group 2 consisting of 110 patients with segmental kyphosis. All patients underwent instrumented PLF at the same medical institute, and the average follow-up period was $31 \pm 19$ months. We obtained plain radiographs of the lumbosacral spine with the anteroposterior view, the lateral view, and the dynamic flexion-extension views before the operation and during the follow-ups. The results of PLF in the two groups were then compared.

Results: $\quad$ There was no significant difference in the demographic data of the two groups, except for gender distribution. The osseous fusion rates were $90.7 \%$ in group 1 and $68.2 \%$ in group $2(p<0.001)$.

Conclusion: Instrumented PLF resulted in significantly higher osseous fusion rate in patients without segmental kyphosis than in the patients with segmental kyphosis. For the patients with

At a Glance Commentary

Scientific background of the subject

Treatment by posterolateral fusion with pedicle-screw instrumentation can be unsuccessful in one-segment and low-grade lumbar spondylolisthesis. Segmental kyphosis, either rigid or dynamic, was demonstrated to be one of the factors interfering with the fusion results.

\section{What this study adds to the field}

For the patients with low-grade spondylolisthesis and segmental kyphosis, such as rigid or dynamic kyphosis, pedicle-screw fixation cannot ensure successful posterolateral fusion. Interbody fusion by the posterior lumbar interbody fusion or transforaminal lumbar interbody fusion technique might help overcome this problem.

sagittal imbalance, such as rigid or dynamic kyphosis, pedicle-screw fixation cannot ensure successful PLF. Interbody fusion by the posterior lumbar interbody fusion or transforaminal lumbar interbody fusion technique might help overcome this problem.

(Biomed J 2015;38:262-268)

Key words: anterior column stability, lumbar spondylolisthesis, posterolateral fusion, segmental kyphosis

P pondylolisthesis is an anterior subluxation or displace$\checkmark$ ment of one vertebral body in relation to another, such as subluxation of the caudal vertebra in the sagittal plane. ${ }^{[1]}$ According to the Wiltse classification system, spondylolisthesis is classified into five types based on the causes: Congenital, degenerative, isthmic, traumatic, and pathologic. ${ }^{[2]}$
In Taiwan, the prevalence of lumbar spondylolisthesis is $6 \%$ in women and $3 \%$ in men. ${ }^{[3]}$ Spondylolisthesis is one of the most common conditions for which surgery is performed ${ }^{[4,5]}$ however, conservative management remains the treatment of choice until the patients develop progressive or disabling symptoms, intractable backache along the spinal axis, or

From the Department of Orthopaedic Surgery, Chang Gung Memorial Hospital at Linkou, Chang Gung University College of Medicine, Taoyuan, Taiwan

Received: Aug. 21, 2014; Accepted: Oct. 24, 2014

Correspondence to: Dr. Chi-Chien Niu, Department of Orthopaedic Surgery, Chang Gung Memorial Hospital at Linkou. 5, Fusing St., Geuishan, Taoyuan 333, Taiwan. Tel: 886-3-3281200 ext. 2163; Fax: 886-3-3284564; E-mail: niuchien@adm.cgmh.org.tw

DOI: $10.4103 / 2319-4170.145768$ 
significant neurological deficits. Surgical treatment is performed to achieve neurological decompression, maintain the lumbar stability, and correct the sagittal and coronal alignment of the vertebral column. Surgical alternatives include posterior decompression, posterolateral fusion (PLF) with or without instrumentation, ${ }^{[6-11]}$ and interbody fusion. ${ }^{[12,13]}$

However, the ideal surgical approach remains debatable in routine practice. Although PFL with pedicle-screw instrumentation is a well-accepted method for treating lumbar instability, certain factors are thought to interfere the fusion by this method, and a range of clinical fusion rates have been reported in the literature. ${ }^{[14-24]}$ Bridwell et al observed more successful and higher fusion rates in the group with pedicle-screw fixation than in that without any instrumentation. ${ }^{[7]}$ However, McClain et al observed a high rate of failure of the hardware, especially when the anterior instability was left untreated. ${ }^{[18]}$ Suda et al concluded that in the treatment of isthmic spondylolisthesis by PLF with pedicle-screw instrumentation, factors such as preserved disc height and segmental kyphosis influence the fusion and lead to pseudoarthrosis or instrument breakage; $;^{[21]}$ they hypothesized that segmental kyphosis, rigid or with flexion, represents anterior column insufficiency and is one of the factors interfering with the fusion.

In this study, we recruited 239 patients with single-segment, low-grade lumbar spondylolisthesis with or without segmental kyphosis. All the patients were treated by PLF with pedicle-screw instrumentation without interbody fusion in our institution between January 2004 and December 2005. In this retrospective study, we collected and compared the radiographic and clinical results of these patients.

\section{METHODS}

From 2004 to 2005, before the introduction of the interbody cage system at our facility, 259 patients with single-level spondylolisthesis and PLF with pedicle-screw instrumentation were recruited. Of these, 20 were excluded because of pedicle-screw malposition, incomplete clinical data, and inadequate follow-up periods. Thus, 239 patients ( 77 men and 162 women; average age, $57.1 \pm 12.7$ years) with the average follow-up of $31 \pm 19$ months remained for the final analysis. The etiology was degeneration in 187 patients $(78 \%)$ and isthmus spondylolytic type in 52 patients (22\%). In $149(62.3 \%)$ of the patients, spondylolisthesis was located over the L4/L5 levels; it was also located at the L3/L4 level (11 patients, $4.6 \%$ ) or L5/S1 level (42 patients, 18\%).

The inclusion criterion for the study was as follows: Single-segmental lumbar spondylolisthesis with grade I or II slippage based on the Meyerding system, ${ }^{[25]}$ which was treated by PLF with pedicle-screw instrumentation and autogenous bone grafting. None of the patients were treated by interbody fusion. Informed consents were obtained from all the patients before performing the operations, and all of them were followed up for at least 1 year after the operation.

In total, 259 patients met the inclusion criterion. However, patients with hardware complications (such as pedicle-screw malposition), inadequate durations of postoperative follow-up (less than 1 year), and incomplete clinical data were excluded from the study. Twenty patients were excluded: 17 because of incomplete clinical data or an inadequate duration of follow-up, 2 because they had undergone re-operation for the removal of malpositioned pedicle-screws, and 1 for undergoing re-operation for adjacent instability within 1 year of the operation.

We performed transverse process decortication and intertransverse process bone grafting by using local bone chips obtained by laminectomy and autogenous iliac bone grafts. Taillard's method was adopted for the documentation of the slippage degree in the patients on the basis of the results of lateral dynamic radiographs of the lumbar spine. ${ }^{[26]}$ In order to control the magnification bias in the radiographs, we calculated the ratio of the slip distance to the length of the upper endplate of the lower vertebral body. The anterior disc height was measured by determining the length of the perpendicular line from the upper endplate of the lower vertebra over the anterior superior border of the lower vertebra, and the posterior disc height was measured by determining the length of the perpendicular line passing from posterior inferior border of the upper vertebra, as previously detailed. ${ }^{[27]}$

The 239 patients included in this study were divided into two subgroups according to the presence or absence of segmental kyphosis. Group 1 (control group) consisted of 129 patients without segmental kyphosis and group 2 (study group) had 110 patients with segmental kyphosis. We defined segmental kyphosis as a condition where the anterior disc height was less than the posterior disc height within two adjacent vertebrae in the upright and neutral position (rigid kyphosis) or in the flexion position (dynamic kyphosis) [Figure 1]. Segmental kyphosis was considered to represent insufficient anterior column support and was proved to be one of the risk factors leading to instrumentation failure and pseudoarthrosis. ${ }^{[21]}$

We obtained plain radiographs of the anteroposterior and lateral views of the lumbosacral spine and the dynamic flexion-extension views before and 3, 6, 9, and 12 months, and annually after the operation during the follow-ups. An independent orthopedic surgeon who was not involved in the surgeries reviewed all the radiographs.

The fusion results of arthrodesis were judged on the basis of the Lenke classification system, ${ }^{[28]}$ according to fusion mass formations observed on the plain radiographs [Figure 2]. Definite solid fusion was considered to be achieved in the presence of big, bilateral, solid, trabeculated fusion masses. Possible solid fusion was considered to be 
achieved if large, unilateral, fusion masses were detected along with small, contralateral ones. Cases of fusion segments with bilateral, small, and thin fusion masses were considered as probably not solid arthrodesis, and those of fusion segments showing absorption of bilateral fusion masses or obvious pseudoarthrosis were concluded to be definitely not fused. Cases of definite solid fusion and possible solid fusion were considered as adequate arthrodesis, and others were considered to be inadequate arthrodesis.

\section{Data analysis}

The binary logistic regression test was applied for comparing the results of fusion by arthrodesis. The unpaired Student's $t$-test and the Chi-square test were used to compare the continuous and nominal data, respectively. A difference of 0.05 was considered statistically significant. Statistical analysis was conducted by an independent statistician blinded to the surgical outcomes. Statistical analysis was performed by using the SPSS software, version 13.0 (LEAD Technologies Inc.).

\section{RESULTS}

There were no significant differences between the two groups with respect to the demographic data, except for gender distribution [Table 1]. The percentage of male population was higher in the group without segmental kyphosis (41.8\% in group 1 and $20.8 \%$ in group $2, p<0.05$ ). The anterior column insufficiency was more severe and the disease more advanced in female patients than in male patients.

The surgical outcomes between the study and the control groups were similar, without any significant differences

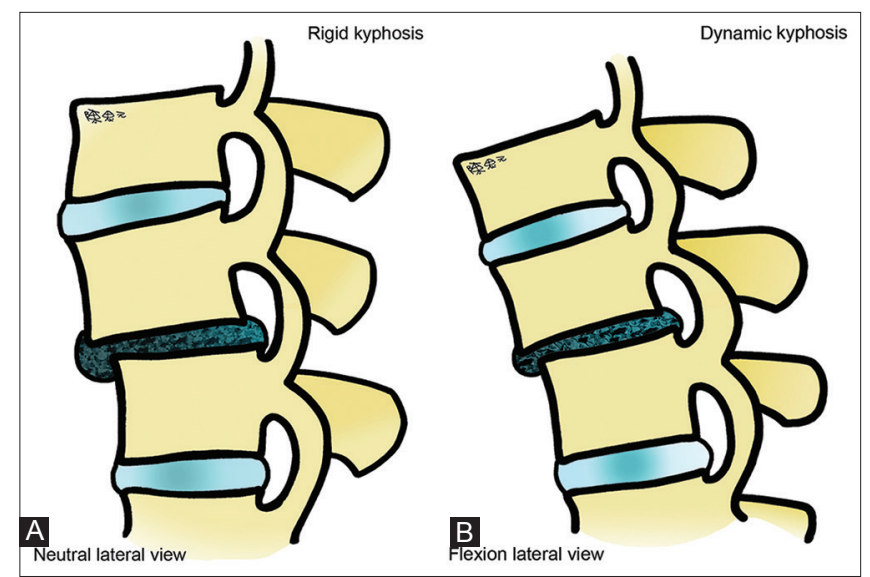

Figure 1: Segmental kyphosis as a result of failed anterior column support, which can be classified into rigid or dynamic kyphosis. (A) In rigid kyphosis, the anterior disc height is less than the posterior disc height in the upright neutral position. (B) In dynamic kyphosis, the anterior disc height is less than the posterior disc height in the forward bending position (dynamic view). in the operative time, blood loss, length of hospital stay, and complication rates [Table 2].

The fusion rates were $90.7 \%$ and $68.2 \%$ in groups 1 and 2 , respectively; the odds ratio between the two groups was 4.5 (95\% confidence interval: $2.2-9.3, p<0.001$ ), which shows a significantly higher arthrodesis rate in patients without segmental kyphosis after they received instrumented PLF [Table 3].

One patient in whom proximal adjacent degeneration was observed 4 years after the operation received revision PLF; acute cervical epidural hematoma compression related to Frankle B neurologic deficit was observed in another patient of group 1 on the first day after the operation. One patient was observed to have distal adjacent degeneration 21 months after the operation, one was observed to have Foley catheter related urethral trauma, one was observed to have superficial wound infection during the second month

Table 1: Demographic data

\begin{tabular}{lccc}
\hline & $\begin{array}{c}\text { Group 1, no } \\
\text { kyphosis }(n=129)\end{array}$ & $\begin{array}{c}\text { Group 2, with } \\
\text { kyphosis }(n=110)\end{array}$ & $p$ \\
\hline Age (years) & $58.5 \pm 13.2$ & $55.3 \pm 12.0$ & 0.076 \\
& $(22-78)$ & $(24-86)$ & \\
Gender (male/female) & $46 / 83$ & $20 / 90$ & $0.002^{*}$ \\
BMI $\left(\mathrm{kg} / \mathrm{m}^{2}\right)$ & $26.3 \pm 3.6$ & $25.9 \pm 4.1$ & 0.509 \\
& $(16.2-35.0)$ & $(19.1-42.3)$ & \\
Smoker & 14 & 5 & 0.070 \\
Nature & $100(78.1)$ & $87(79.1)$ & 0.856
\end{tabular}

( $n, \%$ degenerative)

Level ( $n^{1}, \%$ L3/4; $n^{2}, \% 5$ (4.8); 76 (72.4); 6 (6.2); 73 (75.3); 0.707 $\left.\mathrm{L} 4 / 5 ; n^{3}, \% \mathrm{~L} 5 / \mathrm{S} 1\right) \quad 24(22.9) \quad 18(18.6)$

Data are given as mean values \pm standard deviation with range. ${ }^{*} p<0.05$ was considered statistically significant. Abbreviation: BMI: Body mass index

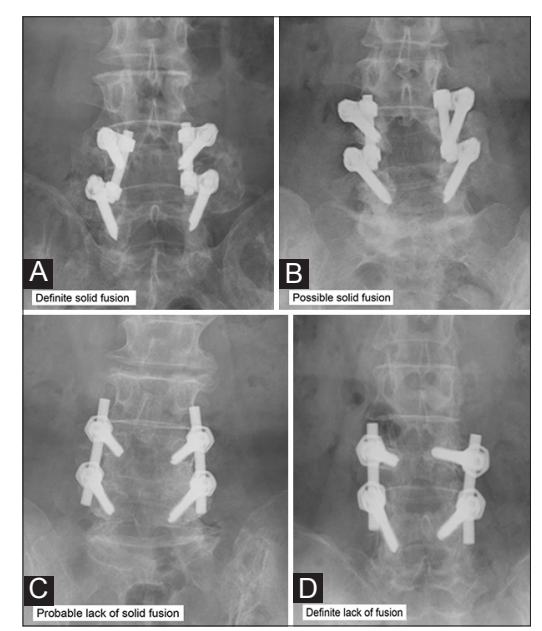

Figure 2: Lenke classification for posterolateral fusion: (A) Definite solid fusion; (B) Possible solid fusion; (C) Probable lack of solid fusion; (D) Definite lack of fusion. (A) Bilateral solid big trabeculated fusion mass. (B) Unilateral large fusion mass along with a contralateral small mass. (C) Bilateral small and thin fusion mass. (D) Absorption of bilateral fusion mass or obvious pseudoarthrosis. 

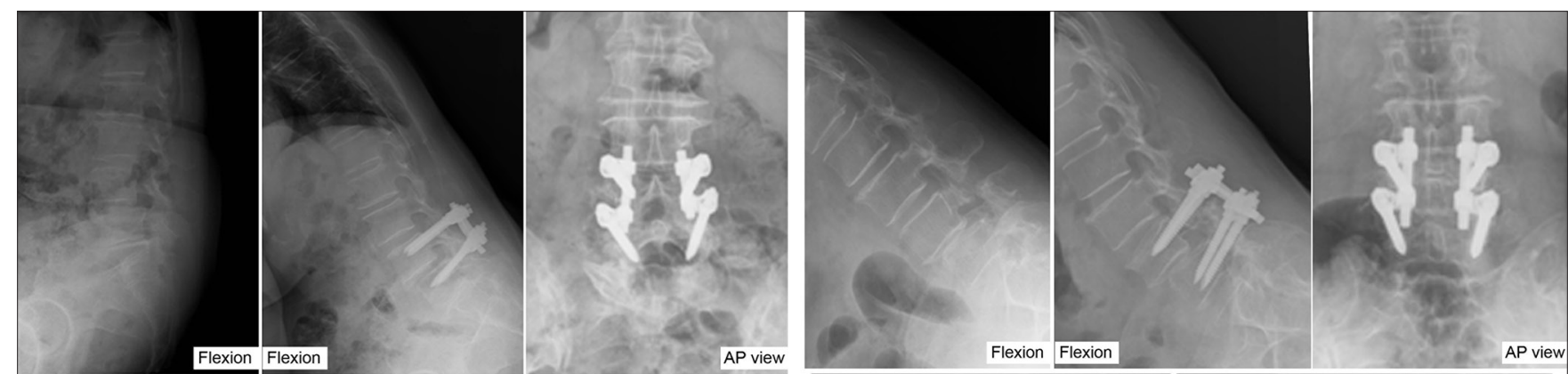

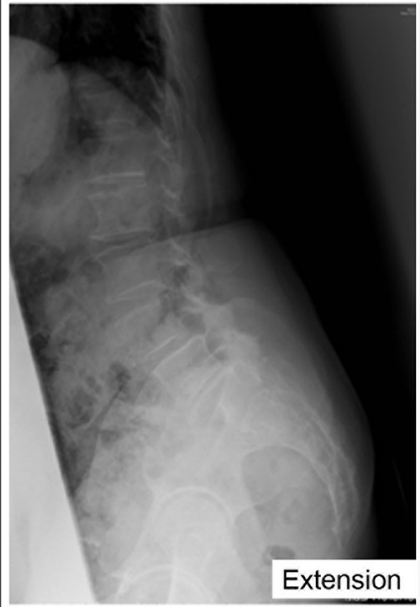

Before surgery

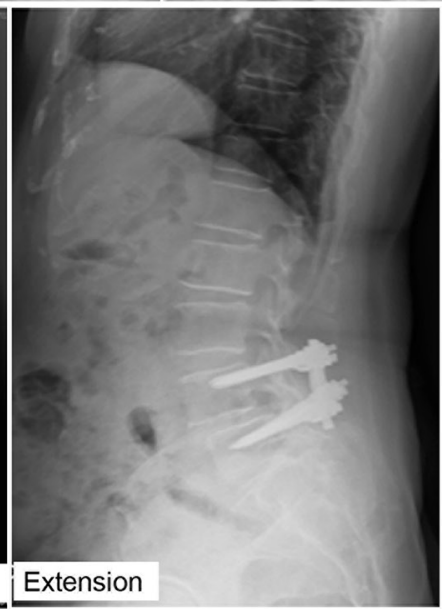

2-year after surgery

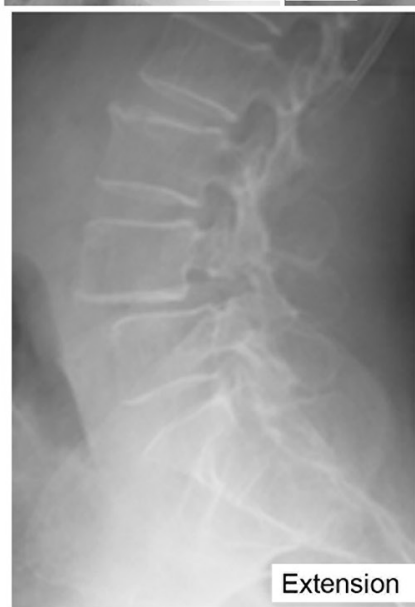

Before surgery

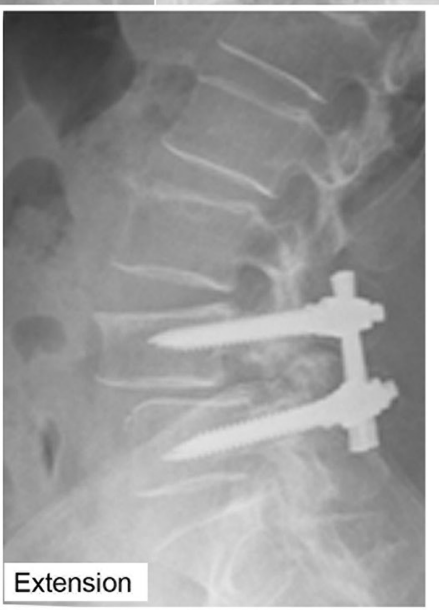

2-year after surgery

Figure 3: Case presentation: (A) A 68-year-old woman's preoperative radiograph showing L4 grade I degenerative spondylolisthesis over L5 (severity of displacement was $22 \%$ and $13 \%$ in the flexion and extension positions, respectively); no rigid or dynamic kyphosis was observed. Laminectomy was performed between the lower half of L4 and the upper half of L5 in addition to L4 and L5 PLF with the Smartloc pediclescrew system and autologous iliac bone grafting. Follow-up radiographs 2 years after the operation showed formation of a big, solid, bilateral fusion mass. (B) Another 55-year-old woman with L4 grade I degenerative spondylolisthesis over L5 (severity of slippage was $27 \%$ and $29 \%$ in the flexion and extension positions, respectively) and obvious rigid and dynamic kyphosis. Instrumented L4 and L5 PLF by Smartloc and autologous iliac bone grafting after adequate nerve decompression were performed. Fusion-graft absorption with bilateral pseudoarthrosis was diagnosed from the follow-up radiographs.

Table 2: Surgical outcome

\begin{tabular}{lccc}
\hline & $\begin{array}{c}\text { Group 1, no } \\
\text { kyphosis }(n=129)\end{array}$ & $\begin{array}{c}\text { Group 2, with } \\
\text { kyphosis }(n=110)\end{array}$ & $p$ \\
\hline Surgical time (min) & $175.9 \pm 39.5$ & $177.9 \pm 35.4$ & 0.711 \\
& $(113-325)$ & $(102-279)$ & \\
Blood loss (ml) & $373.6 \pm 271.4$ & $363.4 \pm 214.8$ & 0.749 \\
& $(50-1300)$ & $(50-1100)$ & \\
Hospital stay (days) & $7.3 \pm 1.6(4-14)$ & $7.3 \pm 1.6(5-15)$ & 0.779 \\
Complication & 2 & 4 & 0.205 \\
\hline
\end{tabular}

Data are given as mean values \pm standard deviation with range. There were no significant differences between the results of the two groups

Table 3: Fusion result

\begin{tabular}{lcc}
\hline & $\begin{array}{c}\text { Group 1, no } \\
\text { kyphosis }(n=129)\end{array}$ & $\begin{array}{c}\text { Group 2, with } \\
\text { kyphosis }(n=110)\end{array}$ \\
\hline Definite and possible fusion & 117 & 75 \\
Probable and definite non-fusion & 12 & 35 \\
Fusion rate & $90.7 \%$ & $68.2 \%$ \\
\hline
\end{tabular}

Odds ratio of group 1/group 2=4.5 (95\% confidence interval, 2.2-9.3, $p<0.001)$. "Logistic regression model, excluding the influence of gender after the operation, and one more patient was observed to have a broken Hemovac drainage tube - all these patients belonged to group 2 .

Two cases with similar severity of slippage presented in Figure 3 were demonstrated to have different instrumented PLF results because of various degree of anterior column stability. Without segmental kyphosis of the affected functional unit, patient of Figure $3 \mathrm{~A}$ had better fusion result than the patient of Figure 3B.

\section{DISCUSSION}

Which surgical approach should be used to treat spondylolisthesis is a debatable issue. ${ }^{[14-21]}$ Instrumented PLF has long been widely accepted as the standard method for vertebral fusion for treating spinal instability and for restoring spinal alignment, ${ }^{[6,7,29,30]}$ but failure of fusion and pseudoarthrosis are common in this method. ${ }^{[15-21]}$ Use of a combination of anterior and posterior fusion in the 1990s 
contributed to improved fusion rates in the surgical treatment of spondylolisthesis..$^{[31,32]}$

After the development of interbody cage fusion, there have been numerous reports on the benefits of the combination of instrumented PLF and interbody fusion for high-grade spondylolisthesis ${ }^{[13,27]}$ owing to their biomechanical advantages and better anterior column supports. ${ }^{[33]}$ Interbody fusion with a cage is beneficial in that the load-bearing capacity of the vertebral column is restored, intervertebral disc height is maintained, intervertebral foramina is distracted, stabilization is immediate, and larger and excellent fusion bed is achieved between the vertebral bodies, thus providing a greater contact area for fusion. ${ }^{[34]}$

However, the need for additional anterior column support for low-grade displacement is debatable since various clinical outcomes have been reported. ${ }^{[32,34-40]}$ Kim et al compared PLF, posterior lumbar interbody fusion (PLIF), and PLIF in combination with PLF in a recent prospective randomized study on degenerative lumbar diseases and concluded that there were no significant differences in the clinical results and union rates. ${ }^{[37]}$ In a study by William et al as well, no consistent differences were noted in the clinical outcomes of groups treated with PLF and PLIF. ${ }^{[35]}$ In a randomized controlled trial conducted by Inamdar et al on a small patient population (11 patients in each group), no incidence of pseudoarthrosis with $100 \%$ fusion rate was observed in patients treated with PLF and PLIF. ${ }^{[40]}$ Dantas et al observed better clinical outcomes and fewer complications in the patients who underwent PLIF in combination with PLF than those who underwent PLF alone. ${ }^{[41]}$ In the study by Müslüman et al, the early clinical outcomes of higher fusion ratio and better clinical results were achieved by concomitant PLF and PLIF treatments. ${ }^{[39]}$ Lei Cheng et al also documented better fusion rates and lower complication rates in the group of patients who underwent PLIF in combination with PLF; however, no significant statistical differences were observed ${ }^{[34]}$ Swan et al found significantly better outcomes with circumferential fusion at 6 months and 1 year postoperatively than the patients who received instrumented PLF; however, the results in the two study groups were similar 2 years after the treatment. ${ }^{[36]}$

These inconsistent clinical results may be attributed to grouping errors. Insufficiency of vertebral anterior column support had not been identified in either group, and preexisting damaged anterior columns can be attributed to the lower fusion rate in the aforementioned studies. Anterior column insufficiency may also have influenced the results of instrumented PLF.

Some authors consider the interbody fusion technique as being disadvantageous because it leads to excessive bleeding and is associated with prolonged operation time, ${ }^{[38]}$ more extensive dissection and dural manipulation, and potential devastating complications such as end-plate erosion, cage displacement with neural component compression, and higher construct stiffness resulting in excessive load on adjacent segments. While these are the potential risks involved in circumferential vertebral arthrodesis, application of interbody cages for fusion results improvement of low-grade spondylolisthesis should have appropriate indications.

Our results suggest that local kyphosis, either rigid or dynamic, can be easily measured in order to quantify the degree of anterior column stability and is an important risk factor contributing to failure of instrumented PLF in the treatment of single-segmental and low-grade spondylolisthesis. In our study, we found that pedicle-screw fixation cannot ensure successful fusion in patients with sagittal imbalance such as rigid kyphosis, or severe disc insufficiency such as dynamic kyphosis. These might be a good indication for interbody fusion cage implantation for additional anterior column support and circumferential fusion.

In the bovine cadaveric study conducted by Oda, ${ }^{[42]}$ instrumented PLF alone was biomechanically adequate when the load-sharing function of anterior column was preserved. If the anterior column is damaged, pedicle-screw fixation alone cannot restore sufficient stability and leads to excessive stress on the posterior instruments with higher instrumentation failure rates. These results are consistent with our findings, thus indicating that either dynamic or rigid kyphosis contributes to statistically low arthrodesis rates. Interbody cages should be considered for increasing segmental stability and avoid instrumentation failure in the case of damaged anterior columns.

Similar results were obtained in the retrospective long-term follow-up study conducted by Suda et al, ${ }^{[21]}$ who concluded that preoperative segmental kyphosis in the upright neutral position leads to instrumentation failure and pseudoarthrosis in the treatment of isthmic spondylolisthesis patients who have received instrumented PLF. Ha et al inferred that interbody fusion maintained the sagittal profile and improved the fusion rates and clinical results in patients with unstable spondylolisthesis, a condition defined as having a degree of slip that was greater than $4 \mathrm{~mm}$ or a slip angle greater than $10^{\circ} .{ }^{[27]}$

Since severity of vertebral translation is a well-known risk factor leading to failure of instrumented PLF, we focused on the patient group with grade I or grade II spondylolisthesis. Damaged anterior column support was quantified as diminished anterior disc height compared with posterior disc height in upright neutral position (rigid kyphosis) or in flexion position (dynamic kyphosis). Both dynamic and rigid kyphosis indicated insufficient intervertebral disc support and proved to lead to inferior osseous fusion results when instrumented PLF alone was performed. In such circumstances, additional anterior column support by using interbody cage may provide adequate stability and help achieve successful circumferential 
fusion. Further comparative studies should be conducted to confirm the effectiveness of instrumented PLF with and without additional interbody fusion either by PLIF or transforaminal lumbar interbody fusion (TLIF) technique for treating low-grade and two-segmental spondylolisthesis by our indications.

\section{Conclusions}

Although PLF with pedicle-screw instrumentation is a well-accepted procedure for the treatment of lumbar spondylolisthesis, anterior column stability is thought to influence the fusion results. Segmental kyphosis, either rigid or dynamic, is believed to be an important risk factor for pseudoarthrosis. Instrumented PLF in patients without segmental kyphosis can help achieve significantly high rate of osseous fusion $(90.7 \%$ ) and is sufficient for the stabilization of spondylolisthesis without segmental kyphosis. Circumferential fusion by using interbody cage is not required for these patients because of the high complication rates and additional costs associated with this technique.

For the patients with sagittal imbalance such as rigid kyphosis, or severe disc insufficiency such as kyphosis with flexion, pedicle-screw fixation cannot ensure successful PLF (fusion rate of $68.2 \%$ ), and interbody fusion by PLIF or TLIF techniques might help overcome this problem.

\section{REFERENCES}

1. Denard PJ, Holton KF, Miller J, Fink HA, Kado DM, Yoo JU, et al. Lumbar Spondylolisthesis among elderly men. Spine 2010;35:1072-8.

2. Wiltse LL, Newman PH, Macnab I. Classification of spondylolisis and spondylolisthesis. Clin Orthop Relat Res 1976;117:23-9.

3. Chen JC, Chan WP, Katz JN, Chang WP, Christiani DC. Occupational and personal factors associated with acquired lumbar spondylolisthesis of urban taxi drivers. Occup Environ Med 2004;61:992-8.

4. Rosenberg NJ. Degenerative spondylolisthesis. Predisposing factors. J Bone Joint Surg Am 1975;57:467-74.

5. Iguchi T, Wakami T, Kurihara A, Kasahara K, Yoshiya S, Nishida K. Lumbar multilevel degenerative spondylolisthesis: Radiological evaluation and factors related to anterolisthesis and retrolisthesis. J Spinal Disord Tech 2002;15:93-9.

6. Herkowitz HN, Kurz LT. Degenerative lumbar spondylolisthesis with spinal stenosis. A prospective study comparing decompression with decompression and intertransverse process arthrodesis. J Bone Joint Surg Am 1991;73:802-8.

7. Bridwell KH, Sedgewick TA, O’Brien MF, Lenke LG, Baldus C. The role of fusion and instrumentation in the treatment of degenerative spondylolisthesis with spinal stenosis. J Spinal Disord 1993;6:461-72.

8. Cunningham BW, Kotani Y, McNulty PS, Cappuccino A, McAfee PC. The effect of spinal destabilization and instrumentation on lumbar intradiscal pressure: An in vitro biomechanical analysis. Spine
1997;22:2655-63.

9. Shono Y, Kaneda K, Abumi K, McAfee PC, Cunningham BW. Stability of posterior spinal instrumentation and its effects on adjacent motion segments in the lumbosacral spine. Spine 1998;23:1550-8.

10. Zdeblick TA. A prospective, randomized study of lumbar fusion: Preliminary results. Spine 1993;18:983-91.

11. Wang PJ, Chen WJ, Chen LH, Niu CC. Spinal fusion and pedicle screw instrumentation in the treatment of spondylolisthesis over the age of 60. Changgeng Yi Xue Za Zhi 1998;21:436-41.

12. Suk SI, Lee CK, Kim WJ, Lee JH, Cho KJ, Kim HG. Adding posterior lumbar interbody fusion to pedicle screw fixation and posterolateral fusion after decompression in spondylolytic spondylolisthesis. Spine 1997;22:210-9.

13. Brodke DS, Dick JC, Kunz DN, McCabe R, Zdeblick TA. Posterior lumbar interbody fusion: A biomechanical comparison, including a new threaded cage. Spine 1997;22:26-31.

14. Boos N, Marchesi D, Aebi M. Treatment of spondylolysis and spondylolisthesis with Cotrel-Dubousset instrumentation: A preliminary report. J Spinal Disord 1991;4:472-9.

15. Boos N, Marchesi D, Zuber K, Aebi M. Treatment of severe spondylolisthesis by reduction and pedicular fixation: A 4-6 year follow-up study. Spine 1993;18:1655-61.

16. Esses SI, Sachs BL, Dreyzin V. Complications associated with the technique of pedicle screw fixation: A selected survey of ABS members. Spine 1993;15:2231-9.

17. Hsu J, Zuckerman JF, White AH, et al. Internal fixation with pedicle screws. In: White AH, Rothman RH, Roy CD, editors. Lumbar Spine Surgery. St Louis: CV Mosby; 1987. p. 322-38.

18. McClain RF, Sparling E, Benson DR. Early failure of short-segment pedicle instrumentation for thoracolumbar fractures. A preliminary report. J Bone Joint Surg Am 1993;75:162-7.

19. Vaccaro AR, Garfin SR. Internal fixation (pedicle screw fixation) for fusions of the lumbar spine. Spine 1995;20 (24 Suppl):157-65S.

20. Knop C, Fabian HF, Bastian L, Blauth M. Late results of thoracolumbar fractures after posterior instrumentation and transpedicular bone grafting. Spine 2001;26:88-99.

21. Suda K, Ito M, Abumi K, Haba H, Taneichi H, Kaneda K. Radiological risk factors of pseudoarthrosis and/or instrument breakage after PLF with the pedicle screw system in isthmic spondylolisthesis. J Spinal Disord 2006;19:541-6.

22. Meyerding HW. Spondylolisthesis: Surgical treatment and results. Surg Gynecol Obstet 1932;54:371-7.

23. Taillard W. Le spondylolisthesis chez l'enfant et l'adolescent. (Etude de 50 cas.) Acta Orthop Scand 1954;24:115-44.

24. Herkowitz HN. Degenerative lumbar spondylolisthesis. Spine 1995;20:1084-90.

25. Muggleton JM, Kondracki M, Allen R. Spinal fusion for lumbar instability: Does it have a scientific basis? J Spinal Disord 2000;13:200-4.

26. Hanley EN Jr. The indications for lumbar spinal fusion with and without instrumentation. Spine 1995;20 (24 Suppl):143-53S.

27. Ha KY, Na KH, Shin JH, Kim KW. Comparison of posterolateral fusion with and without additional posterior lumbar interbody fusion for degenerative lumbar spondylolisthesis. J Spinal Disord Tech 2008;21:229-34. 
28. Lenke LG, Bridwell KH, Bullis D, Betz RR, Baldus C, Schoenecker PL. Results of in situ fusion for isthmic spondylolisthesis. J Spinal Disord 1992;5:433-42.

29. Feffer HL, Wiesel SW, Cuckler JM, Rothman RH. Degenerative spondylolisthesis. To fuse or not to fuse. Spine 1985;10:287-9.

30. Booth KC, Bridwell KH, Eisenberg BA, Baldus CR, Lenke LG. Minimum 5-year results of degenerative spondylolisthesis treated with decompression and instrumented posterior fusion. Spine 1999;24:1721-7.

31. Kim SS, Denis F, Lonstein JE, Winter RB. Factors affecting fusion rate in adult spondylolisthesis. Spine 1990;15:979-83.

32. Huang KF, Chen TY. Clinical results of a single central interbody fusion cage and transpedicle screws fixation for recurrent herniated lumbar disc and low-grade spondylolisthesis. Chang Gung Med J 2003;26:170-7.

33. van Dijk M, Smit TH, Sugihara S, Burger EH, Wuisman PI. The effect of cage stiffness on the rate of lumbar interbody fusion: An in-vivo model using poly (L-lactic acid) and titanium cages. Spine 2002;27:682-8.

34. Cheng L, Nie L, Zhang L. Posterior Lumbar interbody fusion versus posterolateral fusion in spondylolisthesis: A prospective controlled study in the Han nationality. Int Orthop 2009;33:1043-7.

35. Abdu WA, Lurie JD, Spratt KF, Tosteson AN, Zhao W, Tosteson TD, et al. Degenerative spondylolisthesis. Does fusion method influence outcome? Four-year results of the spine patient outcomes research trial. Spine 2009;34:2351-60.
36. Swan J, Hurwitz E, Malek F, van den Haak E, Cheng I, Alamin T, et al. Surgical treatment for unstable low-grade isthmic spondylolisthesis in adults: A prospective controlled study of posterior instrumented fusion compared with combined anterior-posterior fusion. Spine J 2006;6:606-14

37. Kim KT, Lee SH, Lee YH, Bae SC, Suk KS. Clinical outcomes of 3 fusion methods through the posterior approach in the lumbar spine. Spine 2006;31:1351-8.

38. Dantas FL, Prandini MN, Ferreira MA. Comparison between posterior lumbar fusion with pedicle screws and posterior lumbar interbody fusion with pedicle screws in adult spondylolisthesis. Arq Neuropsiquiatr 2007;65:764-70.

39. Müslüman AM, Yilfmaz A, Cansever T, Cavuşoğlu H, Colak I, Genç HA, et al. Posterior lumbar interbody fusion versus posterolateral fusion with instrumentation in the treatment of low-grade isthmic spondylolisthesis: Midterm clinical outcomes. J Neurosurg Spine 2011;14:488-96.

40. Inamdar DN, Alagappan M, Shyam L, Devadoss S, Devadoss A. Posterior lumbar interbody fusion versus intertransverse fusion in the treatment of lumbar spondylolisthesis. J Orthop Surg (Hong Kong) 2006;14:21-6.

41. Dai LY, Jia LS, Yuan W, Ni B, Zhu HB. Direct repair of defect in lumbar spondylolysis and mild isthmic spondylolisthesis by bone grafting, with or without joint fusion. Eur Spine J 2001;10:78-83.

42. Oda I, Abumi K, Yu BS, Sudo H, Minami A. Types of spinal instability that require interbody support in posterior lumbar reconstruction: An in vitro biomechanical investigation. Spine 2003;28:1573-80. 